\title{
Efeito da inclusão de diferentes fontes lipídicas e óleo mineral na dieta sobre a digestibilidade dos nutrientes e os níveis plasmáticos de gordura em equinos ${ }^{1}$
}

\section{Rodrigo Martins Ribeiro², Waleska Tobo Pastori² ${ }^{2}$ Marco Henrique Ramos Fagundes², Ligia Dias Prezotto ${ }^{3}$, Alexandre Augusto de Oliveira Gobesso ${ }^{4}$}

\author{
1 Projeto financiado pela FAPESP. Proc. 04/06404-7. \\ 2 Programa de Pós-graduação em Nutrição e Produção Animal, Faculdade de Medicina Veterinária e Zootecnia, Universidade de São Paulo \\ (FMVZ-USP). \\ 3 Zootecnista. \\ ${ }^{4}$ Faculdade de Medicina Veterinária e Zootecnia, Universidade de São Paulo (FMVZ-USP).
}

RESUMO - Objetivou-se avaliar os efeitos da inclusão de gordura de origem animal ou vegetal e de óleo mineral sobre a aceitabilidade, a digestibilidade dos nutrientes e as concentrações plasmáticas de triglicérides e colesterol em equinos. Utilizaram-se quatro potros de 13 a 16 meses de idade recebendo dieta contendo feno de gramínea e concentrado, em delineamento quadrado latino, analisado por contrastes ortogonais. A aceitabilidade não foi influenciada pela quantidade nem pelo tipo de óleo adicionado às dietas. Os menores valores de digestibilidade de matéria seca (MS), matéria orgânica (MO) e extrato etéreo (EE) foram observados para as dietas acrescidas de óleo mineral (58,90; 60,29 e 32,02\%) em comparação à dieta controle, cujos valores foram de 62,58; 64,41 e 77,72\%. O coeficiente de digestibilidade do EE obtido com a dieta com óleo mineral foi menor (32,02\%) que o obtido para as dietas com gordura animal (90,26\%) e gordura vegetal $(86,47 \%)$. A dieta com óleo mineral reduziu a concentração de HDL-C $(68,75 \mathrm{mg} / \mathrm{dL})$ em relação à dieta controle $(76,00 \mathrm{mg} / \mathrm{dL})$. A adição de fontes lipídicas e óleo mineral não influencia na aceitabilidade da dieta por equinos. O óleo vegetal não se diferencia da gordura animal quanto à digestibilidade dos nutrientes, no entanto, essas fontes de lipídios afetam a digestibilidade do extrato etéreo. A adição de óleo mineral reduz os níveis plasmáticos de HDL-C, ao passo que a adição de gordura animal e gordura vegetal não altera as concentrações plasmáticas de colesterol.

Palavras-chave: aceitabilidade, colesterol, digestibilidade, equídeos, triglicérides

\section{Inclusion of different fat sources and mineral oil in equine diet}

\begin{abstract}
The objective of this study was to assess the effects of including animals or vegetable fats and mineral on the acceptability, nutrient digestibility and triglyceride plasma concentrations and cholesterol in horses. Four foals aged 13 to 16 months were used receiving diet containing grass hay and concentrate. The experimental design was a Latin Square; using orthogonal contrasts. The acceptability was not influenced by the quantity nor the type of oil added to the diets. The smallest dry matter (DM) digestibility values, organic matter (OM) and ether extract (EE) were observed for the diets with the addition of mineral oil (58.90, 60.29 and 32.02\%) compared to the control diet, whose values were 62.58, 64.41 and $77.71 \%$. The EE digestibility coefficient obtained for the diets with animal fat (90.26\%) and vegetable fat $(86.47 \%)$. The diet with mineral oil reduced the HDL-C concentration $(68.75 \mathrm{mg} / \mathrm{dL})$ compared to the control diet $(76.00 \mathrm{mg} / \mathrm{dL})$. The adition of fat sources and mineral oil did not influence the acceptability of the diet by the horses. The vegetable oil did not differ from animal fat regarding nutrient digestibility, but these fat sources affected ether extract digestibility. The addition of mineral oil reduced the plastmatic HDL-C levels while the addition of animal fat and vegetable fat did not alter the cholesterol plasmatic concentrations.
\end{abstract}

Key Words: acceptability, cholesterol, digestibility, horses, triglycerides

\section{Introdução}

O principal benefício na utilização de lipídios na dieta é a maior quantidade de energia fornecida quando já se alcançou a taxa máxima de consumo de matéria seca (Lawrence, 1995).
A adição de lipídios em dietas para equinos em substituição aos carboidratos solúveis tem se mostrado uma prática vantajosa, pois os lipídios produzem efeitos benéficos, melhorando o desempenho em exercícios, treinamentos e competições (McCann et al., 1987). 
A digestão dos lipídios ocorre no intestino delgado e a absorção dos ácidos graxos, assim com a dos triglicerídios e colesterol, ocorre na parte distal do intestino delgado (íleo). Entretanto, os ácidos graxos não absorvidos no intestino delgado podem chegar ao ceco/cólon (Jorgensen et al., 2000).

Segundo Correa \& Correa (1985), as lipoproteínas são constituídas de lipídeos neutros (triglicérides e ésteres de colesterol), fosfolipídios e colesterol associado a lipoproteínas, que são classificadas de acordo com a densidade e incluem lipoproteínas de muito baixa densidade (VLDL-C); de baixa densidade (LDL-C) e de alta densidade (HDL-C). As albuminas não pertencem ao grupo das lipoproteínas e são incluídas como transportadoras de ácidos graxos no sangue.

Várias dietas contendo lipídios podem ser oferecidas a equinos, no entanto, em geral, equinos não aceitam a gordura animal. Outras fontes de lipídios, como o óleo vegetal, são bem aceitas por esses animais. A aceitabilidade também pode ser um problema quando a gordura é oferecida em grande quantidade e em pouco tempo (Geelen et al., 2001). O óleo mineral (parafina líquida, vaselina líquida ou óleo de parafina) é uma mistura de hidrocarbonetos líquidos obtidos do petróleo e, quando administrado por via oral, não é digerido e praticamente não é absorvido pelo organismo. Quando usado por 2 a 3 dias, o óleo reduz a consistência das fezes ou pode recobrir a mucosa intestinal reduzindo a absorção de água (Spinosa, 1999).

O objetivo neste estudo foi avaliar o efeito da inclusão de duas fontes de lipídios, óleo vegetal e gordura animal (óleo de soja e sebo suíno), e os efeitos da adição de óleo mineral na dieta de equinos sobre a aceitabilidade, os coeficientes de digestibilidade dos nutrientes e as concentrações plasmáticas de triglicerídios e colesterol (HDL-C,LDL-Ce VLDL-C).

\section{Material e Métodos}

O experimento foi conduzido nas instalações do Setor de Equideocultura e as análises, no Laboratório de Bromatologia do Departamento de Nutrição e Produção Animal da Faculdade de Medicina Veterinária e Zootecnia da Universidade de São Paulo (FMVZ).

Durante o período experimental, os animais foram alimentados com feno de gramínea Cynodon dactylon (L.) Pers. Var. "Coast cross" e concentrado experimental com a seguinte composição: 25\% de soja extrusada; 60 \% de rolão de milho; $10 \%$ de fubá de milho; $4 \%$ de núcleo mineral; e $1 \%$ de sal comum (Tabela 1), além de sal mineral para equinos e água à vontade, segundo recomendações para potros estabelecidas pelo NRC (1989).
Tabela 1 - Composição nutricional dos componentes da dieta, com base na matéria seca

\begin{tabular}{lcc}
\hline Nutricional (\%) & Concentrado & Feno \\
\hline Matéria seca & 90,37 & 92,64 \\
Extrato etéreo & 4,66 & 1,43 \\
Proteína bruta & 14,02 & 13,85 \\
Fibra em detergente ácido & 12,37 & 38,98 \\
Fibra em detergente neutro & 19,9 & 80,38 \\
Cálcio & 1,9 & 0,29 \\
Fósforo & 0,61 & 0,2 \\
\hline
\end{tabular}

Utilizaram-se quatro potros com 13 a 16 meses de idade e $288 \pm 7,68 \mathrm{~kg}$, pulverizados contra ectoparasitos, imunizados contra tétano e vermifugados. Os animais foram alojados em baias individuais exclusivas, devidamente identificadas e forradas com cama de serragem de madeira. Utilizou-se o delineamento experimental em quadrado latino $4 \times 4$ balanceado, composto de quatro animais, quatro tratamentos e quatro repetições, em três contrastes ortogonais.

As dietas foram compostas de feno e concentrado e diferiram entre si apenas quanto à fonte de gordura, que foi adicionada ao concentrado em quantidade correspondente a 5\% do total da dieta (300 g). As fontes de lipídios utilizadas foram óleo de soja (óleo vegetal), sebo suíno (gordura animal) e vaselina líquida (óleo mineral), adicionados na quantidade de $10 \%$ do concentrado oferecido para cada animal. As dietas foram fornecidas em duas refeições diárias, às 7 h e 19 h, divididas em partes iguais, considerando o consumo diário individual de $2 \%$ do peso vivo, com $50 \%$ de concentrado e $50 \%$ de volumoso, fornecidos em comedouros separados.

Cada período experimental teve 11 dias de duração: 8 para adaptação à dieta e 3 para coleta total de fezes. As sobras dos alimentos foram retiradas diariamente, pesadas, acondicionadas em sacos plásticos e congeladas para posterior análise. Os animais foram pesados no início e ao final de cada período experimental.

Antes do início do período de colheita total de fezes, foi retirada toda a serragem utilizada como cama nas baias. Durante os três dias destinados à coleta, as fezes coletadas foram identificadas individualmente em sacolas de coleta, pesadas diariamente e, do total excretado, após homogeneização, foram retirados 5\%, que foram acondicionados em sacos plásticos e congelados para análise bromatológica e determinação dos teores de matéria seca (MS), matéria orgânica (MO), proteína bruta (PB), extrato etéreo (EE), fibra em detergente neutro (FDN) e fibra em detergente ácido (FDA), conforme descrito por Silva(1998). 
As análises para determinação dos níveis plasmáticos de triglicérides e colesterol HDL-C, LDL-C e VLDL-C foram realizadas em amostras de sangue colhidas antes da primeira refeição da manhã, como sugerido por Marchello et al. (2000), no final de cada fase de colheita de fezes, por punção da veia jugular, com agulhas $40 \times 12$ em frasco de tubo seco. As concentrações do HDL-colesterol e de triglicerídeos foram determinadas pelo método colorimétricoenzimático. O LDL-colesterol e VLDL-colesterol foram calculados, respectivamente, pelas fórmulas [(colesterolHDLcolesterol)-(triglicerídeos/5)] e triglicerídeos/5. Foram também calculadas as razões colesterol/HDLcolesterol eLDL-colesterol/HDL-colesterol.

Os resultados foram analisados pelo programa computacional Statistical Analysis System (SAS, 2001) após verificação da normalidade dos resíduos pelo Teste de Shapiro-Wilk (Proc Univariate) e comparação das variâncias pelo Teste de Hartley (Ott, 1983). Os dados originais foram submetidos à análise de variância para separação das causas de variação, ou seja, efeitos de tratamento, de período e do animal. Os efeitos de tratamento foram separados em três contrastes ortogonais: dieta com óleo mineral vs dieta controle; dieta com gordura animal vs dieta com óleo vegetal; dieta com gordura animal + óleo vegetal vs dieta controle. A análise foi realizada utilizando-se o procedimento General Linear Model (PROC GLM do SAS) com nível de significância de 5\% para a metodologia de contraste ortogonal.

\section{Resultados e Discussão}

Não foi observada diferença quanto à aceitabilidade entre as dietas, o que está de acordo com relatos de Holland et al. (1996), em estudo no qual avaliaram a adição de óleo de soja em dietas para equinos, e de Geelen et al. (2001), que também não observaram variação na aceitabilidade por equinos de uma dieta controle e outra com gordura. Mesmo a adição de óleo mineral, ingrediente com alta possibilidade de resistência à ingestão, não alterou a aceitabilidade da dieta, apesar da quantidade utilizada, uma vez que, segundo Andrade et al. (2002), para o tratamento clínico de distúrbios gastrointestinais em equinos, recomenda-se o fornecimento via oral de óleo mineral na quantidade de $2 \%$ do peso vivo do animal (Tabela 2).

Na comparação entre as dietas com gordura animal e óleo vegetal e entre a dieta com os dois tipos de gordura e a dieta controle, não foi observada diferença significativa nos coeficientes de digestibilidade aparente da MS e MO, o que confirma os dados obtidos por Todd et al. (1995), que não notaram alteração na digestibilidade de uma dieta controle e outra com adição de gordura. Bush et al. (2001) também avaliaram o efeito da gordura dietética sobre a digestibilidade in vitro de nutrientes no líquido cecal de dois pôneis alimentados com dieta controle com alfafa e de uma dieta com incremento energético com óleo de milho. O suplemento energético não afetou a digestibilidade da matéria seca e da matéria orgânica. Do mesmo modo, a adição de óleo mineral na dieta não influenciou na digestibilidade aparente da matéria seca e matéria orgânica em comparação à dieta controle.

A digestibilidade aparente da matéria seca e da matéria orgânica diferiu entre a dieta controle e aquela com óleo mineral, o que pode estar relacionado a duas hipóteses: o óleo mineral envolveu os nutrientes, impossibilitando o contato da bile e das enzimas pancreáticas e impedindo a formação micelar (Lewis, 2000); ou o óleo mineral formou uma película na parede intestinal, diminuindo a absorção dos nutrientes e reduzindo a digestibilidade aparente da MS e MO (ChowdHury, 2006).

A digestibilidade aparente da proteína bruta (PB) foi similar entre as dietas, do mesmo modo como observado por McCann et al. (1987), que concluíram que dietas oferecidas a equinos e suplementadas com gorduras não afetaram a digestibilidade aparente da proteína bruta em

Tabela 2 - Digestibilidade dos nutrientes da dieta

\begin{tabular}{|c|c|c|c|c|c|c|c|c|}
\hline \multirow[t]{2}{*}{ Digestibilidade, \% } & \multicolumn{4}{|c|}{ Dieta } & \multirow[t]{2}{*}{$\mathrm{CV}$} & \multicolumn{3}{|c|}{ Contraste } \\
\hline & Controle & $\begin{array}{c}\text { Óleo } \\
\text { vegetal }\end{array}$ & $\begin{array}{l}\text { Gordura } \\
\text { animal }\end{array}$ & $\begin{array}{c}\text { Óleo } \\
\text { mineral }\end{array}$ & & $\begin{array}{l}\text { Óleo mineral } \times \\
\text { controle }\end{array}$ & $\begin{array}{c}\text { Gordura } \\
\text { animal } \times \\
\text { óleo vegetal }\end{array}$ & $\begin{array}{c}\text { (Gordura } \\
\text { animal }+ \text { óleo } \\
\text { vegetal) } \times \\
\text { controle }\end{array}$ \\
\hline Matéria seca & 62,58 & 62,94 & 64,61 & 58,90 & 4,81 & 0,0136 & 0,1398 & 0,2437 \\
\hline Matéria orgânica & 64,41 & 64,33 & 66,19 & 60,29 & 4,72 & 0,0133 & 0,1683 & 0,4427 \\
\hline Fibra em detergente neutro & 44,31 & 43,72 & 46,36 & 42,73 & 10,95 & 0,3024 & 0,1084 & 0,5680 \\
\hline Fibra em detergente ácido & 50,02 & 48,71 & 51,55 & 49,00 & 6,90 & 0,5012 & 0,0924 & 0,9317 \\
\hline
\end{tabular}

$(\mathrm{P}<0,05)$ 
comparação a uma dieta controle, com 15\% de óleo de milho, $15 \%$ de uma mistura de gordura animal e vegetal e 15\% de parafina. Resende et al. (2004) também forneceram 250, 500 e $750 \mathrm{~mL}$ de óleo de milho e não observaram alteração na digestibilidade de proteína bruta da dieta em equinos.

Por outro lado, Jansen et al. (2000) constataram diferença na digestibilidade de PB com a adição de gordura na dieta de equinos e afirmaram que o resultado poderia estar relacionado ao fato de a ingestão de dieta com maior quantidade de gordura poder reduzir o crescimento das bactérias do intestino grosso, o que diminuiria a quantidade da proteína microbiana fecal, ou ao maior consumo de gordura na dieta, que reduziria a a digestão aparente do intestino delgado, em decorrência do aumento do fluxo do nitrogênio endógeno.

$\mathrm{Na}$ avaliação da digestibilidade aparente do extrato etéreo, verificou-se diferença significativa $(\mathrm{P}<0,0001)$ entre a dieta controle e aquela com óleo mineral, mas o óleo mineral não afetou a digestibilidade aparente do EE da dieta, fato comprovado pela quantidade em gramas de EE digerido das dietas controle e com óleo mineral, respectivamente, 141,9 g e $154,5 \mathrm{~g}$, valores semelhantes que indicam que o óleo mineral não foi absorvido, apenas a gordura da dieta, como observado por Andrade et al. (2002) e Spinosa (1999), que, em pesquisa com equinos, não notaram diferenças na digestibilidade do EE de dietas com adição de óleo mineral.

A digestibilidade aparente do EE não diferiu entre a dieta contendo gordura vegetal e aquela acrescida de gordura animal, o que confirma os resultados obtidos por Meyers et al. (1989), que compararam dietas sem adição de gordura a dietas com adição de gordura animal e não observaram diferença na digestibilidade aparente do EE entre as dietas. Na comparação da dieta com gordura animal e gordura vegetal em relação à controle, verificou-se maior digestibilidade aparente do EE da dieta com gordura animal, contrariando relatos de Kronfeld et al. (2004), que forneceram dietas com gordura vegetal e uma dieta controle para equinos e verificaram que a adição de gordura vegetal não alterou estatisticamente a digestibilidade aparente do extrato etéreo. Do mesmo modo, Webb et al. (1987) adicionaram óleo vegetal na dieta para equinos e não notaram diferenças significativas entre a dieta controle e aquela com óleo vegetal.

O aumento da digestibilidade aparente do EE com adição de gordura animal pode ser explicado pela composição da gordura animal, basicamente ácidos graxos saturados, e pelo fato de a absorção intestinal de fontes saturadas aumentar na presença simultânea de ácidos graxos insaturados, especialmente o oleico, o que denota efeito sinérgico na digestibilidade dos ácidos graxos saturados, provavelmente em razão da maior emulsificação no intestino (Chalupa et al., 2002). Esse mecanismo fisiológico pode justificar os valores observados neste experimento, uma vez que o concentrado utilizado possuía ingrediente rico em gordura vegetal (soja integral extrusada), causando sinergismo com a dieta suplementada com gordura animal.

Não foi observada variação na digestibilidade aparente da FDA e da FDN em nenhum dos contrastes ortogonais avaliados. Esse resultado corrobora os descritos por Bush et al. (2001), que não notaram diferença na digestibilidade da FDN da dieta de pôneis alimentados com dieta contendo gordura animal, óleo de soja ou a mistura das duas gorduras. Por outro lado, Jansen et al. (2000), em pesquisa com dietas para equinos, constataram significativa digestibilidade da fibra bruta quando forneceram dietas com óleo de soja. Julen et al. (1995) atribuíram o aumento relativo da digestibilidade aparente da FDN à remoção dos efeitos bloqueadores da fermentação do amido na digestão de fibra no intestino grosso por substituição da gordura por carboidratos.

As variações nos resultados observados na literatura estão relacionadas às quantidades de gordura utilizadas nos estudos, que influi no consumo dos ingredientes das dietas fornecidas aos animais, inclusive os teores de fibra bruta (Morgado \& Galzerano, 2006).

Os valores de triglicerídios (Tabela 3) observados para as dietas controle, com óleo vegetal, com gordura animal e com óleo mineral não indicaram diferenças significativas entre as dietas, o que confirma informações de Marchello et al. (2000) de que os triglicérides também não variam com a suplementação de gordura vegetal à dieta. Geelen (2001), em trabalho com equinos, também não observou diferenças nos valores de triglicérides obtidos com diversos níveis suplementação das dietas com óleo de soja. Hallebeek (2002), do mesmo modo, não notou alteração nos resultados de triglicérides ao adicionarem 150 g de óleo de palma e de soja em 850 g de ração para equinos.

Os valores plasmáticos de triglicerídios obtidos neste experimento não foram significativos, mas aumentaram com adição de gordura e óleo, fato observado também por Siciliano \& Wood (1993), que, utilizando uma dieta com óleo de soja em comparação a uma controle, observaram que as concentrações plasmáticas de triglicérides e colesterol aumentaram no período de 0, 30, 60 e 90 dias. Esse aumento plasmático tem efeito linear nos lipídeos totais. O aumento na concentração plasmática de lipídeos totais em 
Tabela 3 - Níveis plasmáticos de colesterol, HDL, LDL, VLDL e triglicérides em equinos alimentados com dietas suplementadas com três fontes lipídicas

\begin{tabular}{|c|c|c|c|c|c|c|c|c|}
\hline \multirow[t]{2}{*}{$\mathrm{mg} / \mathrm{dL}$} & \multicolumn{4}{|c|}{ Dieta } & \multirow[t]{2}{*}{$\mathrm{CV}$} & \multicolumn{3}{|c|}{ Contraste } \\
\hline & Controle & $\begin{array}{c}\text { Óleo } \\
\text { vegetal }\end{array}$ & $\begin{array}{l}\text { Gordura } \\
\text { animal }\end{array}$ & $\begin{array}{c}\text { Óleo } \\
\text { mineral }\end{array}$ & & $\begin{array}{l}\text { Óleo mineral } \times \\
\text { controle }\end{array}$ & $\begin{array}{c}\text { Gordura } \\
\text { animal } \times \\
\text { óleo vegetal }\end{array}$ & $\begin{array}{c}\text { (Gordura } \\
\text { animal }+ \text { óleo } \\
\text { vegetal) } \times \\
\text { controle }\end{array}$ \\
\hline Celesterol & 98,75 & 89,25 & 99,75 & 94,75 & 12,02 & 0,5073 & 0,1138 & 0,4204 \\
\hline HDL-C & 76,00 & 73,25 & 74,75 & 68,75 & 13,02 & 0,0229 & 0,5529 & 0,3707 \\
\hline LDL-C & 20,75 & 13,50 & 22,45 & 22,00 & 37,92 & 0,8191 & 0,1380 & 0,5628 \\
\hline VLDL-C & 2,00 & 2,50 & 2,55 & 4,00 & 128,54 & 0,2299 & 0,9742 & 0,6972 \\
\hline TRIG & 10,25 & 17,50 & 15,50 & 22,75 & 96,81 & 0,4804 & 0,7365 & 0,4240 \\
\hline
\end{tabular}

$(\mathrm{P}<0,05)$.

ambos os grupos deve refletir a síntese em excesso de metabólicos no armazenamento de triglicérides. Ressalta-se que, tanto nesse experimento como naquele realizado por Siciliano \& Wood (1993), ambos os grupos de cavalos tiveram balanço energético positivo, com ganho de $1 \mathrm{~kg} / \mathrm{dia}$ peso ao longo do experimento.

Os níveis plasmáticos de colesterol dos animais não diferiram entre as dietas avaliadas neste experimento. No mesmo sentido, Fernandez \& McNamara (1994), em pesquisa com suínos alimentados com dietas contendo semente de palma, óleo de palma, gordura suína, gordura bovina, óleo de oliva e óleo, não identificaram diferenças nos níveis plasmáticos de colesterol. O nível de colesterol aumentou quando fornecida a dieta com gordura animal e reduziu quando fornecida a dieta com gordura vegetal, mas esse aumento não foi estatisticamente significativo. De acordo com Hallebeek (2002), quando uma dieta rica em ácidos graxos saturados é substituída por outra rica em ácidos graxos insaturados, ocorre diminuição na quantidade de colesterol, como relatado por Packard (1999), que descreveu que o aumento do colesterol é ocasionado pela saturação dos ácidos graxos da dieta. Os mecanismos ainda não estão esclarecidos na literatura, mas esse aumento pode ocorrer pela interferência no receptor mediador do LDL. Segundo Garg et al (1989), no homem o aumento do colesterol ocorre pela inibição da síntese endógena do colesterol por inibição da HMG-COa redutase, responsável pela síntese do colesterol, aumentando sua taxa de esterificação.

Os níveis plasmáticos de LDL foram mais altos quando fornecida a dieta com gordura animal, seguida pela dieta com óleo mineral, pela dieta controle e depois pela dieta com gordura vegetal, no entanto a diferença não foi estatisticamente significativa, fato que confirma o relato de Hayes (2001) de que dietas com ácidos graxos insaturados diminuem e dietas com ácidos graxos saturados aumentam o índice de LDL.
Os valores VLDL foram muito próximos entre as dietas, confirmando informações de Marchello et al. (2000), que não observaram variação nos resultados de VLDL obtidos com o fornecimento de dietas com óleo e uma dieta controle durante quatro semanas.

Os valores plasmáticos de HDL foram mais baixos, com diferença significativa, quando fornecida a dieta com óleo mineral. Não foram observados valores significativos entre as demais dietas. Esses resultados foram similares aos encontrados por Geelen et al. (2001), que observaram relação entre o aumento dos níveis de óleo de soja da dieta e o aumento do colesterol total e do HDL e atribuíram esse aumento à maior quantidade de lipídios da dieta e, consequentemente, à maior atividade da lipoproteína lípase (LPL). De acordo com McCann et al. (1987) e Hambleton et al. (1990), no entanto, a adição de gorduras na dieta não altera os níveis de HDL em equinos.

\section{Conclusões}

A adição de óleo vegetal, gordura animal e óleo mineral no nível de $5 \%$ da matéria seca ingerida por dia não interfere na aceitabilidade da dieta por equinos. A adição de gordura animal e óleo vegetal pode melhorar a digestibilidade do extrato etéreo e não influencia na digestibilidade aparente dos demais nutrientes da dieta nem nos valores plasmáticos de colesterol, suas frações e triglicerídeos. A inclusão de óleo mineral pode prejudicar a digestibilidade da matéria seca e da matéria orgânica da dieta e alterar o valor de HDL plasmático em equinos.

\section{Literatura Citada}

ANDRADE, S.F.; CAMARGO, P.L.; VIANNA, L.C. et al. Terapêutica do Sistema Digestivo. In: ANDRADE S.F. (Ed.) Manual de Terapêutica Veterinária. 2.ed. São Paulo: Roca, 2002. p.230. BUSH, J.A.; FREEMAN, D.E.; KLINE, K.H. et al. Dietary fat supplementation effects on in vitro nutrient disappearance 
and in vivo nutrient intake and total tract digestibility by horses. Journal of Animal Science, v.79, n.1, p.232-239, 2001.

CORREA, A.A.D.; CORREA, J.H.R. Bioquímica animal. 2.ed. Lisboa: Fundação Calouste Guibenkian, 1985. 1249p.

CHALUPA, W.; VECCHIARELLI, B.; ELSER, A.E. et al. Ruminal fermentation in vivo as influenced by long-chain fatty acids. Journal Dairy Science, v.3, n.69, p.1293-1301, 2002.

CHOWDHURY, S. Exploring thes cience of laxatives: mechaninsms and modes of action. Nurse Prescribing, v.4, n.3, p.107-112, 2006.

GARG, M.L.; THOMSON, A.B.R.; CLANDININ, M.T. Effect of dietary fish oil on tissue lipid metabolism. In: CHANDRA, R.K. (Ed.) Health effects of fish and fish oils. St. John's: ARTS Biomedical Publishers and Distributors Limited, 1989. p.53-79.

GEELEN, N.J.; BLÁZQUEZ, C.; GEELEN, M.J.H. et al. High fat intake lowers hepatic fatty acid synthesis and raises fatty acid oxidation in aerobic muscle in shetland ponies. British Journal of Nutrition, v.86, n.1, p.31-36, 2001.

HALLEBEEK, J.M. Dietary control of equine plasma triacylglycerols. 2002. 140f. Thesis (Doctor in Animal Nutrition) - Universidade de Utrecht, Utrecht, 2002. p.91-104.

HAMBLETON, P. L.; SLADER, L. D.; HAMAR, D. W. et al. Dietary fat and exercise conditioning effect on metabolic parameters in the horse. Journal of Animal Science, v.51, n.6, p.1330-1339, 1990 .

HAYES, K.C. The omega-6 versus omega-3 fatty acid modulation of lipoprotein metabolism. American Chemical Society, n.4, p.37-51, 2001.

HOLLAND, J.L.; KRONFELD, D.S.; SKLAN, D. et al. Calculation of fecal kinetics in horses fed hay or hay and concentrate. Journal of Animal Science, v.76, n.7, p.1937-1944, 1996.

JANSEN, W. L.;VAN DER KUILEN, J.; GEELEN, S.N.J. et al. The effect of replacing nonstructural carbohydrates with soybean oil on the digestibility of fiber in trotting horses. Equine Veterinary Journal, v.32, n.1, p.27-30, 2000.

JORGENSEN, H.; GABERT, M.; HEDEMANN, M.S. et al. Digestion of fat does not differ in growing pigs fed diets containing fish oil, rapeseed oil or coconut oil. The Journal of Nutrition, v.130, n.4, p.852-857, 2000.

JULEN, T.R.; POTTER, G.D.; GREENE, L.W. et al. Adaptation to a fat-supplemented diet by cutting horses. Journal of Equine Veterinary Science, v.15, n.10, p.436-440, 1995

KRONFELD, D.S.; HOLLAND, J. L.; RICH, G.A. et al. Fat digestibility in Equus caballus followa first-order kinetics. Journal of Animal Science, v.82, n.6, p.1773-1780, 2004.

LAWRENCE, L.M. Nutrition for competition: preventing weight loss. Equine Veterinary Education, v.7, n.3, p.325-329, 1995.

LEWIS, L.D. Nutrição clínica eqüina: alimentação e cuidados. 2.ed. São Paulo: Roca, 2000. p.710.
MARCHELLO, E.V.; SCHURG, W.A.; MARCHELLO, J.A. et al. Changes in lipoprotein composition in horses fed a fatsupplemented diet. Journal of Equine Veterinary Science, v.20, n.7, p.453-458, 2000.

McCANN, J.S.; MEACHAM, T.N.; FONTENOT, J.P. Energy utilization and blood traits of ponies fed fat-supplemented diets. Journal of Animal Science, v.65, n.4, p.1019-1026, 1987.

MEYERS, M.C.; POTTER, G.D.; EVANS, J.W. et al. Physiologic and metabolic response of exercising horses to added dietary fat. Journal of Equine Veterinary Science, v.9, n.4, p.218-223, 1989.

MORGADO, E.; GALZERANO, L. Utilização de óleos em dietas para eqüinos. Revista Eletrônica de Veterinária REDVET, v.7, n.10, 2006.

NATIONAL RESEARCH COUNCIL - NRC. Nutrient requirements of horses. 5.ed.rev. Washington, D.C.: National Academy Press, 1989. 100p.

OTT, R.L. An introduction to statistical methods and data analysis. Belmont: Wadsworth, 1983. p.730.

PACKARD, J.C. Plasma lipid and lipoprotein metabolism in the 1990s - What we know and what we need to know. In: BETTERIDGE, D.J. (Ed.) Current perspective: lipids. London: Elsevier, 1999. v.13, p.379-390.

RESENDE JR., T.; RESENDE, A.S.C.; LACERDA JR., O.V. et al. Efeito do nível de óleo de milho adicionado à dieta de eqüinos sobre a digestibilidade dos nutrientes. Arquivo Brasileiro de Medicina Veterinária e Zootecnia, v.56, n.1, p.69-73, 2004.

SICILIANO, P.D.; WOOD, C. H. The effect of added dietary soybean oil on vitamin E status of the horse. In: EQUINE NUTRITION PHYSIOLOGY SYMPOSIUM, 1993, Gainesville. Proceedings.. Gainesville: University of Florida, 1993. p.3-4.

SILVA, D.J.; QUEIROZ, A.C. Análise de alimentos: métodos químicos e biológicos. 2.ed. Viçosa, MG: Imprensa Universitária UFV, 1998. 235p.

SPINOSA, H.S. Medicamentos que interferem nas funções gastrointestinais. In: Farmacologia aplicada a medicina veterinária. 2.ed. Rio de Janeiro: Guanabara Koogan, 1999. p.355.

STATISTICAL ANALYSIS SYSTEM - SAS. SAS user's guide: statistics. Versão 8. Cary: Institute SAS, 2001. 943p.

TODD, L.K.; SAUER, W.C.; CHRISTOPHERSON, R.J. et al. The effect of level of feed intake on nutrient and energy digestibilities and rate of feed passage in horses. Journal Animal Physiology Animal Nutrition, v.73, p.140-148, 1995.

WEBB, S.P.; POTTER, G.D.; EVANS, J.W. Physioogy and metabolic response of race and cutting horses to added dietary fat. In: EQUINE NUTRITION PHYSIOLOGY SYMPOSIUM, 10. 1987, Fort Collins. Proceedings... Fort Collins: 1987, p.115. 